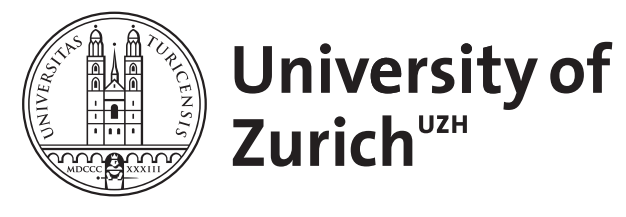

Zurich Open Repository and Archive

University of Zurich

University Library

Strickhofstrasse 39

CH-8057 Zurich

www.zora.uzh.ch

Year: 2009

\title{
Potassium and hypertension
}

Suter, Paolo M

\begin{abstract}
A recent meta-analysis supports the premise that increased potassium intake may play a role in the prevention and treatment of hypertension. The possible mechanisms by which potassium may act as an antihypertensive and recommendations regarding increasing potassium intake are also discussed
\end{abstract}

DOI: https://doi.org/10.1111/j.1753-4887.1998.tb01741.x

Posted at the Zurich Open Repository and Archive, University of Zurich ZORA URL: https://doi.org/10.5167/uzh-154815

Journal Article

Published Version

Originally published at:

Suter, Paolo M (2009). Potassium and hypertension. Nutrition Reviews, 56(5):151-153.

DOI: https://doi.org/10.1111/j.1753-4887.1998.tb01741.x 


\begin{abstract}
A recent meta-analysis supports the premise that increased potassium intake may play a role in the prevention and treatment of hypertension. The possible mechanisms by which potassium may act as an antihypertensive and recommendations regarding increasing potassium intake are also discussed.
\end{abstract}

High blood pressure represents one of the major modifiable risk factors for coronary heart disease, congestive heart failure, stroke, and renal disease. Approximately 20\% of the U.S. adult population has hypertension, which is defined as systolic blood pressure $>140 \mathrm{~mm} \mathrm{Hg}$ and/or diastolic blood pressure $>90 \mathrm{~mm} \mathrm{Hg}$. The prevalence of hypertension varies strongly within and between populations, and there is a profound increase in prevalence rates with increasing age.

The differences in prevalence rates between certain population groups and age groups may be due, in part, to differences in the occurrence of overweight, alcohol consumption, salt intake, physical activity, smoking, and other potentially modifiable risk factors. ${ }^{1}$ The modification of these risk factors represents the cornerstone of any nonpharmacologic strategy of blood pressure control.

The importance of electrolytes in the pathogenesis of hypertension is well known from the blood pressure-lowering effect of salt restriction observed in salt-sensitive individuals. There is a strong interrelationship and interdependence between the electrolytes potassium and sodium in the body. Accordingly, it is not surprising that many epidemiologic and intervention studies have reported a blood pressure-lowering effect of potassium.

Recently, Whelton et al. ${ }^{2}$ performed a meta-analysis of 33 randomized controlled clinical trials to assess the effects of oral potassium supplements on blood pressure. These studies included about 2600 subjects (age range 18-79 years), and the potassium supplementation was the only difference between the intervention groups and control groups. Potassium supplementation was associated with a significant reduction in mean $(95 \%$ confidence interval) systolic and diastolic blood pressure of $3.11 \mathrm{~mm}$ $\mathrm{Hg}(1.91$ to $4.31 \mathrm{~mm} \mathrm{Hg})$ and $1.97 \mathrm{~mm} \mathrm{Hg}(0.52$ to $3.42 \mathrm{~mm}$ $\mathrm{Hg}$ ), respectively. These blood pressure-lowering effects of potassium were especially pronounced in subjects with a high sodium intake. These effects were observed in both subjects who did and subjects who did not take antihypertensive medication. The conclusion of Whelton et al. ${ }^{2}$

This review was prepared by Paolo M. Suter, M.D., M.S., Medical Policlinic, University Hospital, 8091 Zürich, Switzerland. is in agreement with other recent publications on this issue, suggesting that low potassium intake may play an important role in the pathogenesis of hypertension and that increased potassium intake should be considered as a part of treatment of essential hypertension.

In view of this confirmatory meta-analysis on the effect of potassium on blood pressure, several important questions need to be addressed. What is the potential mechanism of potassium's effect on blood pressure? Who can benefit most from increased potassium intake? Should physicians prescribe potassium supplements to patients? Are potassium supplements safe? How can we improve dietary intake of potassium from foods? Detailed discussion of all of these issues is beyond the scope of this review, but some important aspects of the metabolic processes that may explain the role of potassium in blood pressure regulation will be addressed.

It is not known by which mechanism potassium affects blood pressure. Because of the heterogeneity of potassium's effects on different cell and body functions, the blood pressure-lowering effects seem to be the result of several different mechanisms. These include effects on natriuresis, baroreflex sensitivity, direct vasodilatatory functions, catecholaminergic functions, and the central nervous system, as well as improvement of glucose tolerance and modulation of the renin-angiotensin-aldosterone system. ${ }^{3,4}$

As demonstrated in earlier studies, subjects in the Whelton et al. ${ }^{2}$ study with a high sodium intake seemed to have the greatest potential for experiencing blood pressure-lowering effects of potassium. Several epidemiologic studies have described an inverse relationship between potassium intake and blood pressure and a direct relationship between the urinary sodium/potassium ratio and blood pressure. Increased potassium intake is associated with a natriuretic effect and may have a diuretic effect as well. Potassium depletion enhances the urinary loss of calcium, which is another important cation in the regulation of blood pressure. ${ }^{5}$ The increased loss of calcium in this situation may further stimulate parathyroid hormone secretion, which may additionally contribute to an increase in blood pressure. These effects are mediated by the renin-angiotensin-aldosterone system. Potassium depletion leads to increased plasma renin activity via effects on the renal receptors and macula densa. Plasma renin activity can be lowered in most cases by the administration of potassium. Some studies reported a decline in blood pressure of more than $10 \mathrm{~mm} \mathrm{Hg}$ upon potassium supplementation; ${ }^{6,7}$ however, others found no significant effect., ${ }^{8,9}$

The lack of a blood pressure-lowering effect of potassium may be explained by a low-sodium diet, since a 
low sodium delivery to the distal nephron hardly affects potassium secretion and, thus, loss in the urine. Furthermore, in the case of a large natriuretic effect of a higher potassium intake, a lower plasma volume may result, leading to a stimulation of plasma renin activity, which then abolishes the favorable blood pressure effects of potassium.

The so-called baroreceptors are stretch receptors located in some large arteries. Potassium has been reported to influence baroreceptor sensitivity favorably. ${ }^{4}$ In addition, potassium may function as a direct modulator of vascular resistance via its action as a vasodilatator. The vasodilatatory effects of potassium also seem to be of importance in exercise-induced blood pressure changes. During exercise, potassium is released from muscle cells, leading to an increase in extracellular potassium concentration, which induces a vasodilatation that increases local blood flow. Peripheral resistance, and thus blood pressure, may be influenced profoundly. Another action that is dependent on the concentration of potassium and sodium is the uptake of catecholamines from the synaptic cleft. Animal experiments have shown the pressor response to catecholamines could be partially blunted by pretreatment with potassium.

Glucose intolerance, another important risk factor for cardiovascular disease, is a well-known feature of potassium depletion, and it is hypothesized that the disadvantageous effects of potassium-wasting diuretics on glucose metabolism may be mediated by hypokalemia. Accordingly, it is possible that minor changes in potassium balance and status may induce changes in glucose tolerance that lead to changes in blood pressure. It is probably the interplay of all of these mechanisms by which potassium causes a reduction in blood pressure.

Potassium may have many other cardioprotective effects, ranging from inhibition of free radical formation to an effect on platelet aggregation. ${ }^{3}$ Increased potassium concentrations reduce free radical production at the level of the endothelial cell, leading to a favorable blood pressure effect. The inhibitory effects of potassium on vascular smooth muscle cell proliferation may have further protective effects on the development of high blood pressure. These are just a few selected effects of the many important functions of potassium. Other effects include interrelationships between potassium and magnesium and insulin. ${ }^{10}$ Magnesium seems to play an important role in the maintenance of different cardiovascular functions. In magnesium deficiency, potassium wasting occurs in the kidneys. High insulin levels enhance sodium reabsorption and stimulate the sympathetic nervous system.

Clinical experience suggests that the higher the blood pressure, the higher the benefit of any therapeutic intervention. Subjects with mild to moderate hypertension may benefit most from nonpharmacologic means of blood pres- sure control. Some studies have suggested a more pronounced effect of potassium on blood pressure in African Americans. In the meta-analysis of Whelton et al., ${ }^{2}$ the race-related effects of potassium on blood pressure are less clear and less pronounced owing to the ecologic nature of the analysis and the limited information about the racial origin of the participants in some of the studies. Nevertheless, it has been suggested that in some population subgroups, such as African Americans, potassium intake may be even more crucial for blood pressure control than sodium. ${ }^{2,10}$ Furthermore, the benefit seems to be greatest in subjects with borderline hypertension and a positive family history of hypertension.

It is important to note that a high-potassium diet not only should be implemented after the development of hypertension, but also can be beneficial in the normotensive state, and should be a must in the high-normal range of blood pressure. In addition, as with any other strategy for blood pressure control, high potassium intake should occur over a prolonged period of time, presuming there is normal kidney function. The intake of certain antihypertensive medications does not preclude an individual from consuming a high-potassium diet as well.

It is generally not necessary to prescribe potassium supplements, except in the clinical setting of hypokalemia. Because potassium supplements may have severe adverse effects, over-the-counter potassium supplements are not recommended for the general public. A careful promotion of potassium-containing salt-replacement seasonings could be useful; however, the composition of the electrolyte content in different commercial products is highly variable. Carefully reading food labels is very important, because some products contain considerable amounts of sodium, which would counteract the effects of potassium.

Although we do not yet know the physiology of the interaction between the different electrolytes and their effects on different aspects of the cardiovascular system, it is probably acceptable to promote a diet high in potassium. The average potassium intake in the United States varies widely between 30 and $100 \mathrm{mmol} / \mathrm{day} .{ }^{3} \mathrm{In}$ the absence of disease, the body can maintain short-term potassium balance with intakes as high as $10 \mathrm{mmol} / \mathrm{kg}$ body weight. " Most of the potassium is excreted in the urine. Thus, it is not surprising that hyperkalemia is typical in subjects with renal insufficiency. Accordingly, toxicity to high potassium intakes from the diet and/or supplements is a typical feature of subjects with renal insufficiency.

It should be noted that renal insufficiency represents one important endpoint of hypertension. Many hypertensive subjects may have undiagnosed renal insufficiency. Hypertensive subjects on potassium-sparing diuretics represent another high-risk group for hyperkalemia, which can have fatal consequences from cardiac complications. 
Therefore, it would be unfavorable to recommend potassium supplements for all hypertensive subjects or to promote potassium supplements on a population scale, since a major percentage of patients show renal impairment.

Potassium balance is influenced mainly by potassium intake and potassium excretion. Intake is basically a matter of dietary choices. The potassium content of food may vary widely; however, milk, fruits, grain products, vegetables, and even some meats represent good sources. The ratio of dietary sodium to potassium should be taken into account. Food processing can profoundly affect this ratio. For example, raw potatoes have a low sodium/potassium ratio, whereas potato chips have a very high ratio. Legumes, vegetables, fruits, and grains generally have a low ratio. A mainly vegetarian diet provides larger amounts of potassium. It has been suggested that the rather high potassium content of vegetarian diets may be partly responsible for the beneficial blood pressure effects observed in vegetarians. ${ }^{12}$

The recently published Dietary Approaches to Stop Hypertension (DASH) trial ${ }^{13}$ compared the effect of a typical U.S. diet (low in fruits and vegetables) with a diet that was high in fruits and vegetables and, consequently, higher in potassium. The consecutive blood pressure effects were up to $5.5 \mathrm{~mm} \mathrm{Hg}$ systolic and $3.0 \mathrm{~mm} \mathrm{Hg}$ diastolic. The DASH study does not permit us to identify any single nutrient responsible for this significant decline in blood pressure but, rather, an overall dietary pattern. Evidence suggests, however, that increased potassium intake may have been of considerable importance. The consumption of a diet rich in fruits and vegetables is in line with the current Dietary Guidelines for Americans and has been associated with lower rates of chronic disease. It is also a safe and realistic approach to increase potassium intake.

In conclusion, potassium seems to have antihypertensive effects, but no single mechanism has been identified. Because potassium's effects on blood pressure may be related to its complex interaction with several nutrients and substances, no single treatment strategy is successful for all subjects. The blood pressure-lowering effects of potassium may be not experienced by all subjects. Blood pressure should not, however, be the only endpoint for the recommendation of a high-potassium diet. Potassium may have other health effects in the cardiovascular system and may even play a direct or indirect role in carcinogenesis. ${ }^{3,14}$ For the prevention and/or the nonpharmaco- logic therapy of hypertension, potassium from dietary sources (i.e., a diet rich in vegetables and fruits) should be encouraged rather than potassium supplements. Finally, it should be remembered that the control and prevention of hypertension start in the normotensive state and that no single healing electrolyte or nutrient seems to exist. ${ }^{10}$

1. Moore TJ, McKnight JA. Dietary factors and blood pressure regulation. Endocrin Metab Clin North Am 1995;24:643-55

2. Whelton PK, He J, Cutler JA, et al. Effects of oral potassium on blood pressure: meta-analysis of randomized controlled clinical trials. JAMA 1997;277:1624-32

3. Young DB, Lin H, McCabe RD. Potassium's cardiovascular protective mechanism. Am J Physiol 1995;37:R825-37

4. Luft FC, Weinberger MH. Potassium and blood pressure regulation. Am J Clin Nutr 1987;45:1289-94

5. Kristal-Boneh E, Green MS. Dietary calcium and blood pressure: a critical review of the literature. Public Health Rev 1990;18:267-300

6. Siani A, Strazzullo P, Russo L, et al. Controlled trial of long-term oral potassium supplements in patients with mild hypertension. Br Med J 1987;294:14536

7. limura O, Kijima T, Kikuchi K, et al. Studies on the hypotensive effect of high potassium intake in patients with essential hypertension. Clin Sci 1981;61:77s-80s

8. - Grimm RH, Neaton JD, Elmer PJ, et al. The influence of oral potassium chloride on blood pressure in hypertensive men on a low sodium diet. $\mathrm{N}$ Engl $\mathrm{J}$ Med 1990;322:569-74

9. Zoccali C, Cumming AM, Hutcheson MJ, et al. Effects of potassium on sodium balance, renin, noradrenaline and arterial blood pressure. J Hypertens 1985;3:67-72

10. McCarron DA. A consensus approach to electrolytes and blood pressure. Hypertension 1991;17(suppl 1):l-170-2

11. Tannen RL. Hypo-hyperkalaemia. In: Davison AM, Cameron JS, Grünfeld J-P, et al, eds. Oxford textbook of clinical nephrology, vol 1. Oxford: Oxford University Press, 1998;201-24

12. Ophir O, Peer G, Gilad J, et al. Low blood pressure in vegetarians: the possible role of potassium. Am $\mathrm{J}$ Clin Nutr 1983;37:755-62

13. Appel LJ, Moore TJ, Obarzanek E, et al. A clinical trial of the effects of dietary patterns on blood pressure. N Engl J Med 1997;336:1117-24

14. Jansson B. Potassium, sodium, and cancer: a review. J Environ Pathol Toxicol Oncol 1996;15:65-73 